\title{
Embedded Web Services for Industrial TCP/IP Services Monitoring
}

\author{
Francisco Maciá-Pérez \\ University of Alicante \\ Technology and Computer \\ Science Department \\ PO Box 99, 03080, \\ Alicante, Spain \\ pmacia@dtic.ua.es
}

\author{
Diego Marcos-Jorquera \\ University of Alicante \\ Technology and Computer \\ Science Department \\ PO Box 99, 03080, \\ Alicante, Spain \\ dmarcos@dtic.ua.es
}

\author{
Virgilio Gilart-Iglesias \\ University of Alicante \\ Technology and Computer \\ Science Department \\ PO Box 99, 03080, \\ Alicante, Spain \\ vgilart@dtic.ua.es
}

\begin{abstract}
In this paper we present an approach based on the use of embedded network devices for the deployment of small network services suitable for IT management in industrial and manufacturing environments. The novelty of the proposal resides in the fact that allows to unfold IT services that can be deployed without the need for specialized IT staff, since the services in question have a zero maintenance design philosophy. They are presented under both conventional (client-server) and more open (SOA) standards, more specifically, as Web Services. To illustrate the specific the proposal, we have chosen as an example an embedded device able to monitor the correct operation of the applications and IT services located in the current manufacturing equipment and components.
\end{abstract}

\section{Introduction}

Internet capacity for providing clients with a choice of the most up to date and available consumer goods and at competitive prices is currently requiring the industrial sector to progress from traditional mass production manufacturing paradigms towards models which facilitate mass customization [1].

With these new production models, the customer is no longer considered as a mere entity, external to the manufacturing process itself, but rather, becomes a part of it as an active component, determining the specific characteristics of the desired product.

In order to ensure that these manufacturing models are viable, the manufacturing processes must be fully integrated in the organisation global process map [2]. Although new technologies may help considerably in this aim, it is also true that they require a change of scenario for which not all organisations are completely ready. [3].

One of the main problems faced by these organisations is the emergence of new management tasks deriving from the introduction of complex and innovative technologies in manufacturing levels $[4,5,6]$ with new production machinery incorporating IT elements $[7,8]$, ranging from the simplest, to the most complex. This situation is further aggravated by the fact that organisations run itself into a problem in training their employees or finding professionals with the requisite profile and skills.

Our proposal consists of providing embedded IT management services in physical network devices generally, small sized devices with simple services-, so that, in order to deploy those services, it is enough to select the specific device providing the service, and connecting it to the communications network. The device itself will obtain the minimum information required to activate the initial set up and, once this has been completed, execute the management tasks with minimal human intervention. In addition, since the service is provided from a physical device, it does not set in motion too many additional maintenance tasks relating to the infrastructures providing support for the new IT services.

Obviously, from a functional point of view the services offered by these devices are totally compatible with the traditional network services and therefore their integration and interoperability are ensured. More precisely the services implemented are compatible with standard Web Services and with other more traditional client-server protocols within the scope of systems management such as: telnet, TFTP, HTTP or SNMP.

By way of illustration and with the aim of arguing the motivating of the proposal, in this work we suggest a specific management service: a service for the network service monitoring (NSM) build in the current manufacturing equipment as well as the physical device associated with that service (eNSM device). The goal of this service is to reduce stoppage times in the event of failures and discontinuity in the production process.

The basic function of the service will be to indicate to the eNSM device which equipment and which service or services of that equipments we wish to check in order to assure that they are operating correctly (generally by using an IP address, a port and the expected result) If 
there is any incident the device will register it and report in that respect.

In the following sections we provide a review of the current state of the art of the technologies involved, describing the NSM service, the hardware and software structure of the device in which it is embedded, the application protocol specified and its implementation as Web Service. Finally, the conclusions on the research and our current lines of work are exposed.

\section{Background}

The integration of manufacturing processes in the general organisation process map thus achieving continuity is one of the main goals of the industrial sector [9]. Due to physical and technological limitations, manufacturing processes have not reached the desired level of integration and automation, and in most cases they have to be considered as legacy systems. Until quite recently, integration proposals were centred on traditional automation models based on proprietary protocols situated at a resource level of the eBusiness model as systems external to business processes (Modbus, Profibus, AS-I, FIPIO, DeviceNET, Interbus or Ethernet industrial). These proposals were the first attempts to facilitate their integration with business components using ad-hoc adaptors [10].

With the development of internet and electronic advances, solutions have been proposed based on service paradigms, distributed systems and embedded devices with the computational and communications capacity suitable for environments with hostile physical conditions such as those occurring in industrial atmospheres.

Schneider was one of the first manufacturers of automation and industrial control devices to have incorporated these ideas in its automation apparatus in order to communicate with management applications. This trend is reflected in concepts such as transparent factory [11]. Other manufacturers such as ABB, go somewhat further by raising communication to higher levels of organisation using the Simple Object Access Protocol (SOAP), and incorporating intelligence, selfmanagement and proactivity into its embedded devices [12]. Along the same lines, in [5] the use of Web Services is proposed as a normalised means of accessing functionalities of the devices so that they can be integrated with Enterprise Resource Planning systems (ERP). In [13] is proposed to use these same techniques to raise the level of abstraction of production elements to a business level so that the integration of resources, processes and in general business logic are produced naturally and in a transparent manner within the current business models.

Within the framework of European research projects there are some important initiatives which bear out our interest in this line of research, and which have produced significant results and are progressing towards ServiceOriented Architectures (SOA) and embedded devices in industrial machinery as valid technologies [7, 14].

The scientific community is clearly interested in the use of IT paradigms which have established the present WEB bases as a technological framework which supports the execution of processes associated with production elements.

However, as information technologies continue to inundate production plants increasingly further new associated tasks arise, namely the management of the new services and infrastructures used. These tasks are gaining greater importance as the organisation becomes increasingly reliant on IT, requiring the same levels of robustness and security as in the industrial sector.

The first open standards which attempted to address problems of IT management in a global manner were Simple Network Management Protocol (SNMP) and Common Management Information Protocol (CMIP) [15], proposed by the Internet Engineering Task Force (IETF); both protocols being principally oriented towards network monitoring and control. The main inconvenience of these administration models was their dependence on the platform.

Based on these, and seeking an integration with heterogeneous systems, two principal lines of work arose: an attempt to achieve integration of the systems using the same network management protocol, as is the case of [16] and [17] with the use of Common Object Request Broker Architecture (CORBA); and more ambitiously, to propose a network management protocol which would be independent of the infrastructures. Some of the more extensive proposals include: CORBA/JIDM, specification of the work group Joint Inter-Domain Management (JIDM) [18] of the Object Management Group (OMG) [19]; CIM/WBEM, proposal of the Distributed Management Task Force (DMTF) [20] using techniques oriented towards Computer Integrated Manufacturing (CIM) objects and interoperation using HTTP and XML with Web Based Enterprise Management (WBEM); JMX specification defined by the Java Community Process (JCP) [21] which defines a series of Application Programming Interfaces (API) oriented to Java for network management; and WSManagement specification carried out by various companies in the sector (Sun, Intel, MS, AMD) for the integration of service management systems and resources based on Web Services.

The use of multi-agent systems for computer network management provides a series of characteristics which favour automation and self reliance in maintenance processes [22, 23]. The creation of projects such as AgentLink III, the first Coordinated Action on based on Agents financed by the 6th European Commission Framework Programme [24], is a clear indicator of the considerable degree of interest in research into software agents. 
In [25], a proposal is made for a group of basic operations for a Web Service to be standardised within the management networks as a counterpart to standardisation of the SNMP information model under XML development in other works [26].

As a result of the considerable number of tasks associated with network management as well as its diversity and complexity, the work of maintaining these systems involves a high cost for organisations both in terms of resources and also in terms of time and personnel; added to this are the difficulties inherent in engaging staff with the required skills for addressing this new scenario.

In order to relieve these problems, the current trend in IT management is to use outsourcing as a strategy to recoup investments, ensure the continuous availability of infrastructures and services, and to achieve sufficient levels of quality to enable organisations to keep abreast of a changing environment. However, outsourcing is not usually a valid strategy for handling production environments due to problems raised by security privacy and immediacy.

In areas where automated handling of information and those where several devices are involved, such as industrial processes or domotics, there has been a trend in the development of autonomous management towards architectures designed for services for embedded systems [12, 14]. This final framework includes monitoring systems developed by third parties but residing with the client, who is responsible for their control and management. Along these lines we find proposals such as NAGIOS [27], MON [28], MUNIN/MONIT [29, 30] or nPULSE [31] generic monitoring systems for network services for Linux, with Web interface, highly configurable and based on open code which monitors the availability of network services and applications. The disadvantage of these proposals lies in the complexity of their installation and configuration in environments without qualified system administrators, in addition to the complex systems and infrastructures required for their implementation.

\section{Description of the Monitoring Service}

The Embedded Network Service Monitoring (eNSM) service is a Web Service which enables TCP/IP application active monitoring, using for this purpose Internet standard protocols and service-oriented architectures, which render the service regardless of the administrator's location or the platform used.

The main task of the monitoring service is to check that the services and applications contained in the manufacturing components are operating correctly. A network device (known as an eNSM Device) has been designed for this purpose and in which this functionality has been embedded.
This device is small in size, transparent to existing IT infrastructures and with minimum maintenance required from the system administrators.

The administrator informs the device which of the equipment's services or applications need checking. The eNSM device has sufficient knowledge of each service to carry out this task. This knowledge is included in monitoring software agents displaced to the device. In this way if the device receives a request for monitoring a new service, it will be sufficient to request the adequate monitoring of the agent in order to carry out its work.

The monitoring procedure consists of establishing connections with the services and applications to be monitored by means of its own protocols based on $\mathrm{TCP} / \mathrm{IP}$, analysing the responses to standard requests in search of errors, either in the operation of the protocol itself or in the data received.

The eNSM device represents a basic part of the system. Figure 1 shows a diagram of the main elements and actors involved in the service, together with the existing relation between them. We may synthesise these as: eNSM Device, Manufacturing Components, Discovery Service, NSM Center, NSM Clients and a set of Software Agents.

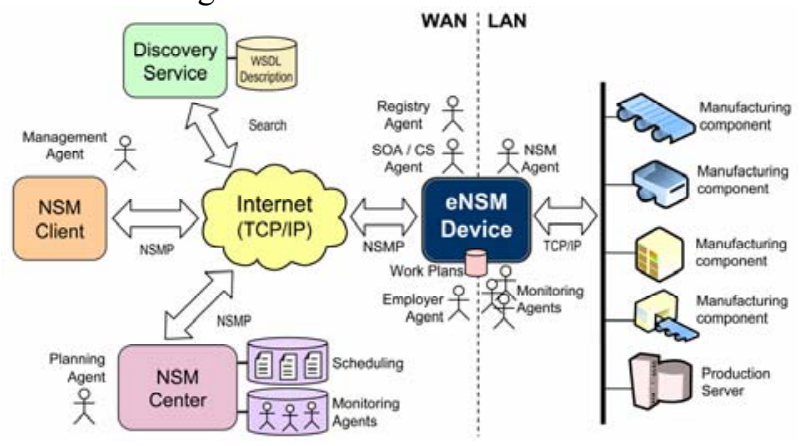

Figure 1. Organization of functional elements of the NSM service.

The eNSM Device, as may be seen, is the cornerstone of the service. It is designed to act as a proxy between the broad area network and the local area network for which it provides support. Instructions from the WAN consist of orders defined in the NSMP protocol which they use SOAP as transport protocol (it is possible to use other options: HTTP, SNMP or telnet). Communications between the device and the manufacturing components of the services and applications to be monitored shall be made directly using native protocols for each of these services or applications.

The Manufacturing Components are the object of the management and comprise all those devices connected to the network and acting as network services containers, which will be monitored.

The Discovery Service comprises a standard UDDI registration service. It is responsible for maintaining the pages describing the NSM services in WSDL format, as 
well as facilitating that information to the clients wishing to access the service.

The NSM Center usually acts as a control panel for all the possible eNSM devices distributed through Internet. This control is implemented through the planning agent who carries out, executes and verifies all the previously established tasks on the eNSM devices. The NSM Centre is also responsible for managing the repository of monitoring agents with the know-how of each monitoring service.

The NSM Client provides the user, through the NSM agent, access to the NSM Center (to manage work plans or query logs files) and to the eNSM Devices (to manage particular services of specifics manufacturing components directly).

The Software Agents consists of a multi-agent system which is actually responsible for executing all the tasks involved in the system. The Register Agents reside in an eNSM Device and undertake to check the monitoring service in a Discovery Service. The Management Agents reside in an NSM Client and are responsible for providing an appropriate interface for the administrators so that they can access the NSM Centre or an eNSM Device from any node connected to Internet. The Planning Agents reside in the NSM Centres and undertake the planning management of eNSM Devices. The SOA Agents and the CS Agents reside in an eNSM Device and represent the SOA interface or client-server, respectively, which compiles the service requests. The NSM Agents are assigned to an eNSM Device and are responsible for coordinating work requests contained in its Work Plan, selecting the appropriate Monitoring Agents for carrying out the work. The Monitoring Agents are stored in the agent repositories managed by the NSM Centres, and they contain the service logic for each type of application or service requiring monitoring, and they are transferred to an eNSM Device, where they carry out the tasks for which they have been programmed. Finally, the Employer Agents are assigned to an eNSM Device and are responsible for locating the Monitoring Agents required by the device to carry out its task.

The sequence diagram in figure 2 shows the basic operation of the service and the multi-agent system. The diagram comprises three blocks and is executed constantly in parallel mode.

In the first block the device interface agents ( $S O A$ Agent and CS Agent) are on standby for requests either from a Planning Agent, or directly from a Management Agent. When they receive a request they attend to it and execute it through the eNSM service kernel. When they receive monitoring requests these are programmed in the Work Plan register of the NSM Agent.

The second block of the diagram corresponds to the execution of the programmed tasks. In this case the NSM Agent is constantly checking the Work Plan on the basis of which it programmes the different Monitoring Agents to carry out the tasks requested.
The third block concerns the contracting of the Monitoring Agents. In blocks 1 and 2, in the event that the monitoring of a service is requested in which the device does not have a Monitoring Agent able to deal with it; both the NSM Agent and the SOA and CS Agents who have detected this lack may make a request to the Employer Agent programming it into its Work Plan. The Employer Agent then undertakes to obtain the Monitoring Agents required by the device. This agent is responsible for negotiating and validating the whole process. The Monitoring Agents are located in the agent repository in the NSM Centre.

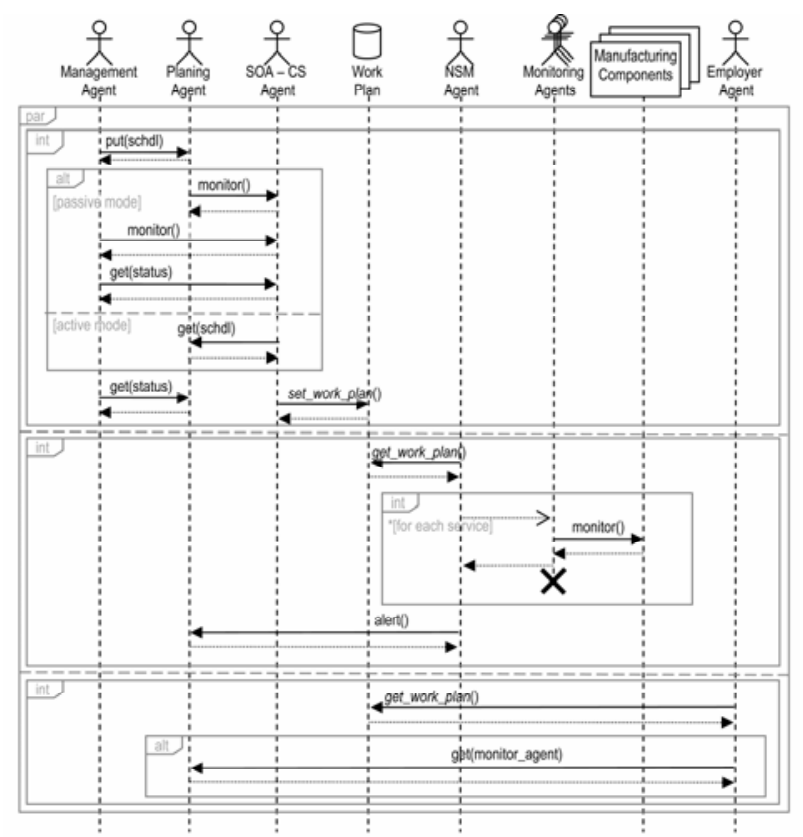

\section{Figure 2. Sequence diagram of the NSM main functionality.}

\section{Monitoring Service Protocol}

The NSM service protocol (NSMP) gathers all monitoring service functionality through a set of instructions. The protocol has defined like a requestresponse text based protocol. This permits that it may become easily adapted to different models, such as client-server (over basic protocols like HTTP, SMTP or telnet) and SOA (over protocols like SOAP).

The syntactic elements for an instruction are:

CMD defines the service actions and corresponds to the name of the remote procedure which implements the functionality of the NSMP command.

ACTION is a special parameter which discriminate the functionality of the request.

ARGS represents the necessary information for executing the $<$ action $>$. 


\begin{tabular}{|c|c|c|c|}
\hline CMD & ACTION & ARG & FUNCTION \\
\hline \multirow[t]{5}{*}{ SET } & \multirow[t]{3}{*}{ MODE } & & Reports the current operation mode. \\
\hline & & PASSIVE [port] & Sets the passive mode and, optionally, the listening port number. \\
\hline & & ACTIVE <ip > [:port] & Sets the active mode, specifying the NSM centerr's IP address and port number. \\
\hline & \multirow[t]{2}{*}{ RUN } & & Reports the current NSM service state. \\
\hline & & $<$ STARTS $\mid$ STOP $>$ & Starts or stops the NSM service. \\
\hline \multirow[t]{2}{*}{ GET } & SCHDL & & Returns the list of scheduled tasks in the device. \\
\hline & STATUS & $\begin{array}{l}{[<\text { host }>[: \text { port }]} \\
[<\text { service }>]]\end{array}$ & Returns the status of a specific service or a set of services. \\
\hline PUT & SCHDL & $<$ schdl-table $>$ & Adds a task or a set of tasks to the scheduling. \\
\hline \multirow[t]{2}{*}{ MONITOR } & ON & $\begin{array}{l}<\text { host }>:<\text { port }><\text { time }> \\
<\text { service }>\text { [arguments] }\end{array}$ & $\begin{array}{l}\text { Establishes a monitoring rule for the address }\langle\text { host }\rangle:\langle\text { port }\rangle \text {, establishing the } \\
\text { poliing time in seconds and the monitor that will be utilized as well as the } \\
\text { arguments that this require. }\end{array}$ \\
\hline & OFF & $\begin{array}{l}<\text { <host }>:<\text { port }> \\
<\text { service }>\end{array}$ & Cancels a monitoring rule. \\
\hline ALERT & & & Send an error alert. \\
\hline
\end{tabular}

\section{Table 1. Main instructions of the NSM protocol}

The main instructions (showed in table 1) that can be embedded in a NSM requests are: SET, GET, PUT, MONITOR and ALERT.

SET command manages the configuration of the internal system variables which determines their function mode. PUT and GET commands, combined with the SCHDL action, program and obtain, respectively, the agents work planning. GET command, with STATUS action, allows getting specific information from the device state.

MONITOR command provides access to the principal service of the device. The instruction syntax to activate a monitoring service is:

MONITOR ON <host> <port> <time> <service> [args] *

As well, in order to disable the monitoring of a service, the instruction format will be:

MONITOR OFF <host> <port> <service>

In two cases, the labels have the following meaning:

$<$ host $>$ is the IP address or the name of the device to monitoring

$<$ port $>$ identifies the service or application whose status wants to verify.

$<$ service $>$ identifies the monitoring agent that must take care of making the analysis of the service.

ALERT Command is designed so that the eNSM device could to notify of the error to the NSM center.

Because is about a request-respond protocol, for each NSMP request there will be a corresponding NSMP response. Basically, this request will be $\mathbf{O K}$, if the order is correctly execute or conversely ERROR if it is not. In some cases the answer can contain more specific information about the realized operation, as GET SCHDL command, which returns the list of tasks programmed.

These instructions must become embedded inside the specific request/response protocol that it be used as transport layer. In the case of choosing a telnet service, instructions and the result of his execution will be literal. In the case of HTTP, the instructions will have to go inserted in a HTTP request body. In strategies to support
Service-oriented architecture, in particular, Web Services, it has utilizing SOAP as mechanism of messages interchange. This case is a little more complex and it requires some more attention.

In SOAP request Document style and RPC style are supported. In the case of Document style each NSM instruction is embedded in the body of a SOAP message which contains the NSMP document, which implements the functionality of the command and the arguments required for its execution. The syntax of NMSP is defined in his correspondent DTD.

In the SOAP messages of RPC style, will exist a single operation (EXECUTE) whose single argument is a text string that will contain the instruction NSMP, of very similar form to as it makes in client-server cases.

\section{Device Design}

In the same way as it has been described previously, the monitoring service is part of a broadest system in which the eNSM device is the cornerstone. The eNSM device combines hardware and software in order to deploy all its functionality, achieving the different requirements that have been established before.

For this reason, a device general architecture has been defined (figure 3). This device architecture is layer-based organized and has a widely accepted structure for embedded devices. In the lower layer the device hardware has been defined, based on a computational system including microprocessor, volatile memory (for the execution), non volatile memory (for the stored system) and communication module for its connection to the network.

In addition of these basic components, it is pertinent to highlight the absence of mechanic elements (as hard disks) and the inclusion of auxiliary elements (as watchdog mechanism or Power over Ethernet supply).

On the hardware layer is placed the embedded operative system. In order to satisfy industrial environment specific requirements - where the device 
will be included and will provide support - real time operative systems has been chosen. The following layer contained the middleware platform to provide services to the applications.

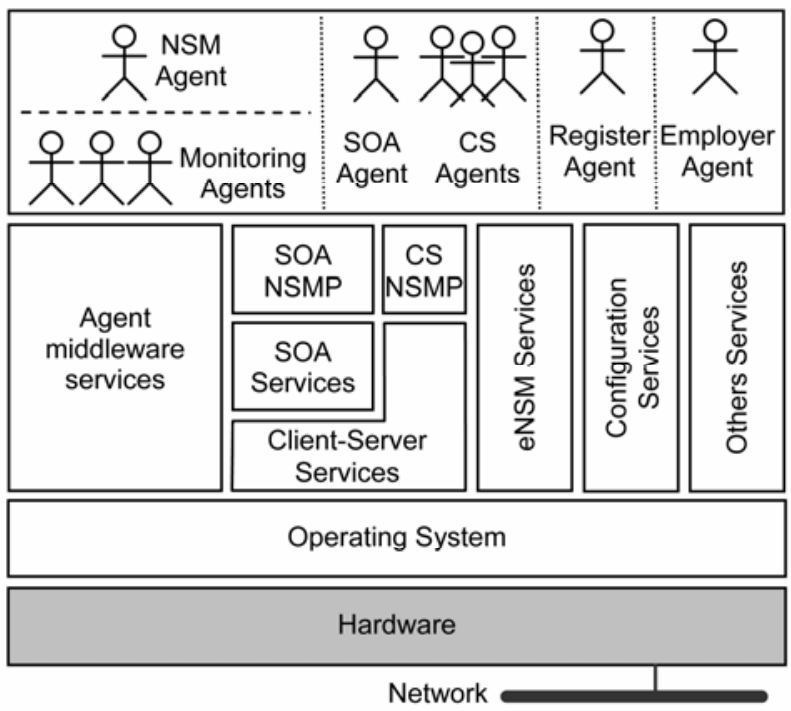

Figure 3. eNSM device architecture.

The first two important elements in this layer comprise different network services, commonly used in the management field, under the client-server model and services oriented architectures (SOA). These modules give basic services so the device can communicate, at the application level, with other external components. In order to make suitable this communication to the syntax of monitoring service instructions, the NSMP protocol is implemented on these modules (in both SOA and CS versions). One of the other important services placed in this layer is the core which contains all the procedures offered by the NSM services. In the same layer is located the middleware platform in order to provide support to the service software agents. These agents are placed in the last layer (application layer) and really, they take charge of provide the service: acting as interface with other services and applications, registering the service in a discovery service, coordinating the monitoring service or, simply, monitoring selected services.

\section{Device Implementation}

In this section the implementation of a prototype of eNSM device is presented taking into account the general architecture exposed in the previous section and specifying the different structural blocks according to the available technologies. In figure 4 the resulting architecture has been given shape graphically.

The hardware platform chosen for the prototype development is a Lantronix Xport ${ }^{\circledR} A R^{\mathrm{TM}}$ device which has a 16 bit DSTni-EXTM processor with $120 \mathrm{MHz}$ frequency reaching 30MIPS respectively (in figure 6 a picture of an eNSM device prototype connected to the service network can be observed ). The various memory modulates provided by this device undertake specific tasks according to their intrinsic features: the execution programmes and the dates handled by the device SRAM memory it resides in the $(1,25 \mathrm{MB})$; the ROM memory (16KB) holds the system start up application and, finally, the flash memory, with $4 \mathrm{MB}$, stores information which though non-volatile, is susceptible to change, such ace the Sep up of the eNSM device or the system applications which may be updated. These capacities are sufficient for the memory requirements of the software developed for implementing the protocol.

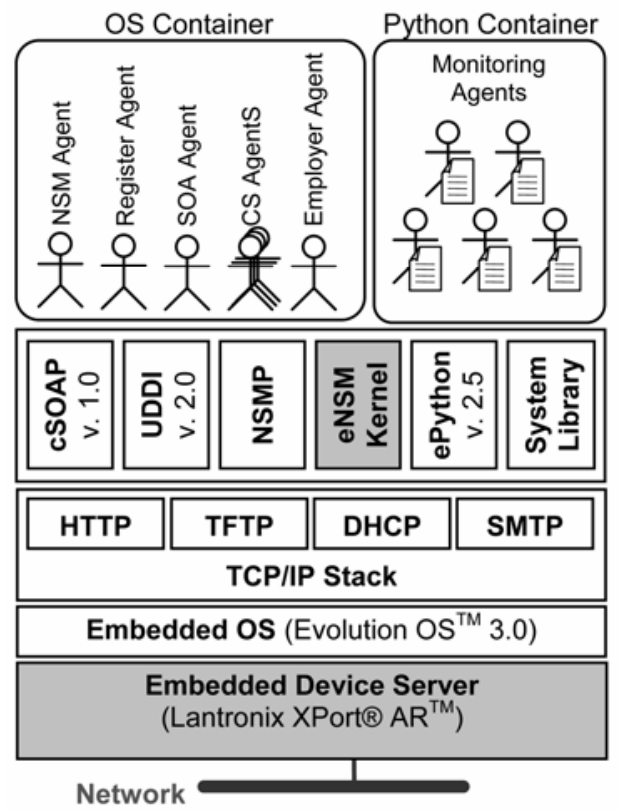

\section{Figure 4. Architecture of the eMSM device prototype.}

Among other I/O interfaces, the device has a Fast Ethernet network interface which allows suitable external communications ratios. In addition, in order to ensure the correct system operation there are several auxiliary elements such as: a watchdog which monitors the CPU and prevents it from blocking; and a PLL frequency divider required to set up the frequency of the system clock with an adjustable clock signal (CLK) to optimise consumption or performance according to needs.

As real time operating system, the device incorporates the version 3 of the Lantronix OS, Evolution OS ${ }^{\mathrm{TM}}$. Through an confidentiality agreement with Lantronix, we have had access to the different modules of the system. Given the space restrictions, this has been crucial to develop a made-to-measure version of this OS. As main elements to point out, in this version, a TCP/IP stack has been included together with several clientserver application protocols (HTTP, TFTP, SNMP and telnet). 
In the service layer, the implementation process has been conditioned by the characteristics of XPort device. Although, with the current hardware miniaturization level it has been enlarged their computational capacities, the devices continue presenting important limitations in their resources.

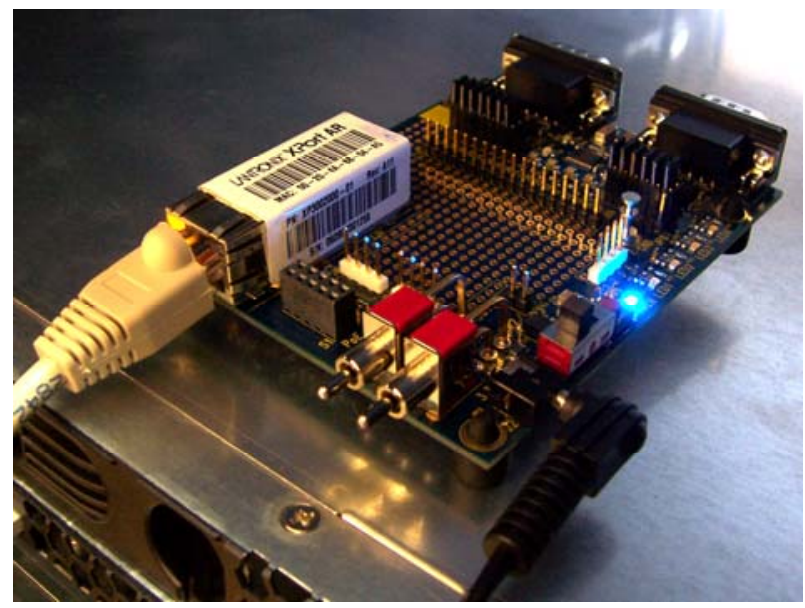

Figure 5. An eNSM Device prototype.

In this layer, three services blocks are implemented: the middleware that provides the communication mechanisms of the monitoring service, the NSM service kernel with the implementation of NSM instructions and the middleware platform that provides the execution of software agents.

The communication service middleware is upheld by standard protocols and technologies included in the Evolution $O S$. In the CS based NSMP implementation, a $\mathrm{C}$ module has been developed to provide a protocol syntactic analyser. In the SOA based NSMP implementation, development has been done using the cSOAP library, which is appropriate for these devices [32]. However, some changes have been done in the original cSOAP library due to device limitations (restriction of memory use, proprietary libraries, etc.). These limitations have forced us to replace cSOAP XML parser, LibXML2 (more than $1 \mathrm{MB}$ in size), by another adapted XML parser with limited but enough functionalities to achieve our objective. Due to cSOAP limitations, only RPC style that use the same protocol analyser used in the client-server version has been developed.

Besides, in order to registry and to publish the services, an UDDI embedded version has been implemented based in UDDI version 2.0 which simply permits publishing the WSDL document associated with the monitoring service. Figure 6 shows a fragment of the WSDL page with the definition of the RPC procedure for the EXECUTE command.

The NSM service kernel has been implemented as a functions library write in C language and offered as API for the others eNSM device modules. By means of this library, the monitoring service intrinsic functionalities are achieved.

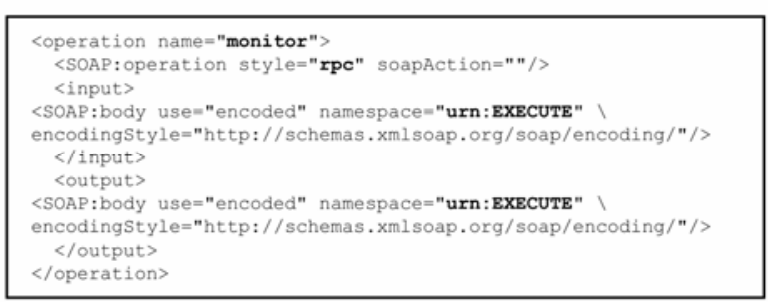

\section{Figure 6. WSDL document fragment for MONITOR command (RPC style).}

In order to implement service agents, a division in the implementation process between static and mobile agents has been realized. In the first case, an ad-hoc implementation for the XPort device has been development in $\mathrm{C}$ language, using operative system as agents' container. In the second case, in order to establish an execution framework for the monitoring agents, a Python embedded engine (ePython version 2.5) has been adapted to the XPort features. These monitoring agents are implemented as Python text scripts.

\section{Conclusions}

In this paper we have presented a system for the provision of IT services directed to manage applications and embedded services in machinery and components of production in the industry. The proposal aims to provide a reference frame in which we could design and implement this kind of service in a systematic way. One of the most relevant aspects of this system consists of providing these embedded management services in network devices. The devices must be adapted to the characteristics of the production and IT environments: small size, simple, low power consumption, adjusted costs, autonomous, designed with safety criteria and robustness, and compatible with the traditional network services through the standard protocols such as: SOAP, SMTP or HTTP. With the aim to validate the proposal, a concrete service has been designed and implemented in order to monitoring the IT embedded services in the current components of manufacture, together with functional prototype of the network device.

We are currently working with other embedded network services and integrating them all in a model based on Semantic Web services, so that in future they will not only be compatible with existing services, but also with new services or setups which were not considered in its initial design.

The final objective of our research is focussed on assuring continuity in the manufacturing processes through technologies that should be as transparent as possible to the users. 


\section{Acknowledgements}

This work was supported by the Generalitat Valenciana under Grant GV/2007175 and Office of the Vice President for International Relations \& Cooperation of the University of Alicante.

\section{References}

[1] C. Younghwan, K. Kwangsoo, K. Cheolhan. "A design chain collaboration framework using reference models," International Journal of Advanced Manufacturing Technology. 26 (1) pp. 183-190. July, 2005.

[2] L. Avella y D. Vázquez. Is Agile Manufacturing a New Production Paradigm? Universia Business Review, ISSN 1698-5117, No 6, pp. 94-107, 2005.

[3] P. Harmon, M. Rosen, M. Guttman. Developing Ebusiness Systems and Architectures: A Manager's Guide. Morgan Kaufmann Publishers, San Francisco, USA. 2001.

[4] D.C. McFarlane and S. Bussmann. "Developments in Holonic Production Planning and Control.” Intenational Journal of Production Planning and Control, Vol. 11, No. 6, pp. 522 -- 536, 2000.

[5] A.P. Kalogeras, J.V. Gialelis, C.E. Alexakos, M.J. Georgoudakis, S.A. Koubias. "Vertical integration of enterprise industrial systems utilizing web services", IEEE Transaction on industrial informatics. VOL II. $\mathrm{n}^{\circ} 2$, pp. 120-128. 2006.

[6] J.L.M. Lastra, M. Delamer. "Semantic web services in factory automation: fundamental insights and research roadmap", IEEE Transaction on industrial informatics. VOL II. $n^{\circ} 1$, pp. 1-11. 2006.

[7] F. Jammes, H. Smit. "Service-Oriented paradigms in industrial automation", IEEE Transaction on industrial informatics. VOL I. n ${ }^{\mathrm{o}}$ 1, pp. 62-70. 2005.

[8] S-M. Lee et al. "A component-based distributed control system for assembly automation", Proceedings of 2nd International Conference on Industrial Informatics. INDIN 2004.

[9] A.P. Kalogeras, J.V. Gialelis, C.E. Alexakos, M.J. Georgoudakis, S.A. Koubias. "Manufacturing Collaborative Process Integration Utilizing State of the Art Technologies" Proceedings of the IEEE International Symposium on Industrial Electronics, ISIE 2005. 2005.

[10] R.P. Moreno. Ingeniería de la automatización industrial. Ra-Ma, Madrid, Spain, 2004.

[11] Transparent Factory. Manual de usuario y planificación. [Online] Available: http://www.modicon.com, 2001.

[12] U. Toop, P. Muller, J. Konnertz, A. Pick, "Web based Service for Embedded Devices," LNCS vol. 2593, 2002, pp. 141-153.
[13] V. Gilart-Iglesias, F. Maciá-Pérez, J.A. Gil-MartínezAbarca and A. Capella-D'alton. "Industrial Machines as a Service: A model based on embedded devices and Web Services". Proceedings of 4th International IEEE Conference on Industrial Informatics (INDIN'06). Singapore, 2006.

[14] F. Jammes, H. Smit, J.L. Martinez-Lastra, I.M. Delamer. "Orchestration of Service-Oriented Manufacturing Processes," Proc. of the 10th IEEE International Conference on Emerging Technologies and Factory Automation, ETFA 2005, Catania, Italy, September 1922, 2005.

[15] RFC Project: http://www.rfc.net (URL).

[16] Jeong, M.S., Kim, K.H., Kwon, J.H., Park, J.T."CORBS/CMIP: Gateway Service Scheme for CORBA/TMN Integration. Knom Review, Vol.2, No. 1, pp. 55-62, 1999.

[17] Aschemann, G., Mohr, T., Ruppert, M. "Integration of SNMP into a CORBA- and Web-Based Management Environment"in Proc. Kommunikation in Verteilten Systemen, , Heidelberg, 1999, pp. 210-221.

[18] Work Group JIDM: http://www.opengroup.org (URL).

[19] OMG: http://www.omg.org (URL)

[20] DMTF: http://www.dmtf.org (URL).

[21] JCP: http://www.jcp.org (URL).

[22] T.C.Du, E.Y. Li, and A.P. Chang, "Mobile Agents in Distributed Network Management", in Communications at the ACM, 46(7), 2003, pp127-132.

[23] J. Guo,Y. Liao, and B. Parviz. "An Agent-Based Network management system", presented at the 2005 Internet and Multimedia Applications.

[24] European co-ordination action for agent-based computing: http://www.rfc.net (URL).

[25] J. Sloten, A. Pras, and M. Van Sinderen, "On the standardisation of web service management operations", presented at the 2004 X EUNICE Summer School and IFIP WG 6.3 Workshop.

[26] T. Klie, and F. Straub, "Integrating SNMP agents with XML-based management systems," IEEE Communications Magazine vol. 42 Issue 7, July 2004, pp 76-83.

[27] NAGIOS: http://nagios.org (URL).

[28] MON: http://www.kernel.org/software/mon/ (URL).

[29] MUNIN: http://munin.projects.linpro.no/ (URL).

[30] MONIT: http://www.tildeslash.com/monit/ (URL).

[31] nPULSE: http://www.horsburgh.com/h_npulse.html (URL).

[32] V. Miori, L. Tarrini, and R. Bianchi, "LIGHT: XMLInnovative Generation for home Networking Technologies", Ercim News, Number 62, July2005. 